\section{Optical coherence tomography, frequency-doubling technology, and colour Doppler imaging in ocular hypertension}

M Cellini, B Bernabini, M Carbonelli, E Zamparini and EC Campos

\begin{abstract}
Purpose To study in ocular hypertension $(\mathrm{OH})$ the retinal nerve fibre layer (RNFL) with optical coherence tomography (OCT) and the neuronal function with frequency-doubling technology (FDT) to assess which of the two methods was more sensitive in detecting early glaucomatous damage. Furthermore, a colour Doppler imaging (CDI) of the optic nerve was carried out to highlight any correlation with RNFL thickness and FDT abnormality. Materials and methods We enrolled 28 ocular hypertensive patients who underwent OCT of the RNFL and FDT. Moreover, we performed a CDI of the ophthalmic artery (OA), central retinal artery (CRA), and posterior ciliary arteries (PCAs).
\end{abstract}

Results The patients with $\mathrm{OH}$ following OCT revealed a significant thinning in the RNFL as compared to the control group only in the inferior quadrant: $122.250 \pm 14.091$ vs $131.750 \pm 10.729 \mu \mathrm{m}(P<0.045)$. As regards FDT, there was a significant difference between the two groups only for pattern standard deviation (PSD): $3.873 \pm 1.488$ vs $1.938 \pm 0.704 \mathrm{~dB}$ $(P<0.044)$. In $\mathrm{OH}$ and in the control group, CDI resistance index $(\mathrm{RI})$ in the OA was $0.768 \pm 0.012$ vs $0.745 \pm 0.019(P<0.022)$, in the CRA was $0.66 \pm 0.012$ vs $0.645 \pm 0.019$ $(P<0.032)$, and in PCAs was $0.673 \pm 0.039$ vs $0.622 \pm 0.012(P<0.037)$. The OCT had a sensitivity of $83 \%$ but only in the inferior RNFL quadrant. The FDT-PSD revealed a sensitivity of $85 \%$.

Conclusions Both FDT and OCT detect early glaucomatous damage with a slightly superior sensitivity of FDT vs OCT. The CDI measurements suggest that circulatory abnormalities may have a role in the development of OCT and FDT damage. Eye (2007) 21, 1071-1077; doi:10.1038/sj.eye.6702412; published online 4 August 2006

Keywords: optical coherence tomography; frequency-doubling technology; colour Doppler imaging; ocular hypertension; retinal nerve fibre layer

\section{Introduction}

Glaucoma is a disease characterized by an increase in intraocular pressure (IOP) with the progressive loss of ganglion cells that causes the onset of optic nerve disorders and consequent visual field alterations. The appearance of alterations in the retinal nerve fibre layer (RNFL) represents an early sign of glaucoma damage that precedes the optic nerve changes and peripheral alterations. ${ }^{1,2}$

The quantitative investigation techniques proposed for the study of the RNFL are scanning laser polarimetry (SLP), ${ }^{3,4}$ Heidelberg retinal tomography (HRT), ${ }^{5,6}$ and more recently optical coherence tomography (OCT). OCT is a technique that makes it possible to examine high-resolution cross-sections of ocular tissues using the low coherence interferometry principle $^{7-10}$ and it seems more reliable than SLP and HRT in the study of RNFL. ${ }^{11,12}$ It is particularly important to use high-quality investigation techniques in the study of glaucoma patients to facilitate early detection of functional deterioration owing to the irreversible nature of the retinal ganglion cell loss and axonal damage. ${ }^{13}$ The qualitative
Department of Surgery and Transplant-

Ophthalmology Service,

'A. Valsalva', University of

Bologna, Bologna, Italy

Correspondence: M Cellini Department of Surgery and TransplantOphthalmology Service, 'A. Valsalva', University of Bologna, Via Massarenti, 9, Bologna I-40138, Italy

Tel: + 3951 341450;

Fax: + 3951342516

E-mail: mauro.cellini@unibo.it

Received: 6 April 2005 Accepted in revised form: 2 April 2006 Published online: 4 August 2006 
analysis methods currently available are standard achromatic perimetry (SAP) and the more sensitive short-wavelight automated perimetry (SWAP) $)^{14,15}$ as well as the recent frequency-doubling technology (FDT) perimetry. ${ }^{16-18}$ There is also still some debate as to whether the appearance of neuronal changes and perimetric damage are vascular or mechanical phenomena.

On the basis of this, we studied the RNFL thickness and the perimetric indices in a group of patients with ocular hypertension $(\mathrm{OH})$ using OCT and FDT to assess which of the two methods was more sensitive in detecting early glaucomatous damage. Furthermore, the patients underwent colour Doppler imaging (CDI) of the ophthalmic artery (OA), central retinal artery (CRA), and the posterior ciliary arteries (PCAs) to highlight any correlations between RNFL thickness, perimetric damage, and haemodynamic changes.

\section{Materials and methods}

A total of 28 patients were studied, 16 men and 12 women, aged between 48 and 65 years (mean 57.4 years) with $\mathrm{OH}$. $\mathrm{OH}$ is diagnosed when the IOP is greater than $21 \mathrm{mmHg}(25.8 \pm 3.1 \mathrm{mmHg})$ with a normal optic disk (in particular with no sign of thinning of the neuroretinal rim, haemorrhage, cupping, or visible changes in the fibre layer) and a value of the perimetric indices MD and CPSD of less than $1.5 \mathrm{~dB}$. All the patients were recruited from the Glaucoma Service of the S. Orsola-Malpighi hospital.

The control group consisted of 28 subjects, 14 men and 14 women aged between 50 and 62 years (mean 54.8 years). Healthy controls were recruited among visitors and staff of the Ophthalmology Service of the S OrsolaMalpighi hospital. The healthy controls enrolled did not have any ongoing or anamneses of eye disorder. The IOP was less than $21 \mathrm{mmHg}$ with normal optic nerve and visual field indices.

Only one eye per subject, both in the $\mathrm{OH}$ group and in control group, was randomly chosen if both eyes were eligible for the study.

The study was approved by the institutional ethic committee of the S Orsola-Malpighi hospital. Before enrolment, patients were informed of the procedures and the aim of the study following which they signed a written consent form.

All the patients underwent an ophthalmologic examination including visual acuity and applanation IOP assessment, corneal radius curvature measurement with automated keratometry (RK, Canon Inc., Tokyo, Japan), corneal thickness valuation with a Tomey SP3000 pakimeter (Tomey Corp., Nagoya, Japan), and biomicroscopy of the anterior and posterior segment with automatic measurement of the $C / D$ area ratio of the optic nerve head with OCT3 (Zeiss-Humphrey, Dublin, CA, USA). We also performed SAP with a Humphrey Field Analyzer-30.2 full-threshold program (ZeissHumphrey, San Leandro, CA, USA). We performed three visual field tests on each $\mathrm{OH}$ patients and the healthy controls and only the third testing was assessed. The demographic and ocular characteristics of the $\mathrm{OH}$ patients and healthy controls are presented in Table 1.

All the subjects underwent an assessment of the RFNL with OCT3 (Zeiss-Humphrey, Dublin, CA, USA), FDT perimetry with RFA (Zeiss-Humphrey, San Leandro, CA, USA), and CDI of the OA, the CRA, and the PCA's using an AU-580 Asynchronous-Hitachi analyzer with a linear $7.5 \mathrm{MHz}$ probe. For FDT we only assessed the third test, and the FDT and SAP were reviewed separately by two investigators masked to any clinical data.

The latest generation OCT3 (Zeiss-Humphrey, Dublin, CA, USA) was used to assess and measure the thickness of the peripapillar retinal fibre layer. This new diagnostic technique makes it possible to analyse the retinal structures, obtaining in vivo tomographic sections illustrating the histological retinal structure. This instrument exploits low coherence interferometry principles, which separate the retinal structures, measuring the echo delay of the light reflected and retrodiffused by the retinal microstructures. OCT3 makes it possible to obtain scans with an axial resolution of $10 \mu$ and a transverse resolution of $20 \mu$. The instrument projects a beam of light on the retina, generated by a superluminescent diode, with a wavelength close to that of infrared $(820 \mathrm{~nm})$. The system detects, processes and memorizes the retinal delay patterns, and displays and memorizes the selected scans so that they can be subsequently analysed. Each eye was dilated with $1 \%$ tropicamide before recording images, and internal fixation was chosen as it provided better reproducibility than external fixation.

Table 1 Comparison of the baseline characteristics among the ocular hypertension and healthy control group

\begin{tabular}{lccc}
\hline & Controls & $\begin{array}{c}\text { Ocular } \\
\text { hypertension }\end{array}$ & $\mathrm{P}<0.05$ \\
\hline Male & 24 & 26 & \\
Female & 24 & 22 & \\
Age (years) & $54.8 \pm 5.2$ & $57.4 \pm 4.8$ & 0.431 \\
Refractive errors (D) & $-0.2 \pm 1.5$ & $-0.4 \pm 1.6$ & 0.320 \\
Corneal radius & $7.764 \pm 0.139$ & $7.829 \pm 0.139$ & 0.210 \\
Corneal thickness & $569.6 \pm 22.57$ & $579.6 \pm 15.25$ & 0.230 \\
C/D area ratio & $0.556 \pm 0.023$ & $0.571 \pm 0.016$ & 0.122 \\
Visual field MD (dB) & $0.28 \pm 1.1$ & $0.31 \pm 1.2$ & 0.670 \\
Visual field CPSD & $0.65 \pm 0.4$ & $0.90 \pm 0.5$ & 0.542 \\
(dB) & & & \\
\hline
\end{tabular}


This instrument was used to measure directly the thickness of the peripapillar RNFL using the RNFL Thickness Average program, which performs three consecutive, circular scans, each one $3.4 \mathrm{~mm}$ in diameter centred around the optic nerve. The thickness of the fibres was defined as the number of pixels obtained between the anterior and posterior RNFL projection.

The values obtained with each scan are shown in graphic form, within a kind of clock face divided into four quadrants, each representing the superior, nasal, inferior, and temporal sections of the RNFL expressed in microns.

FDT perimetry is a new technique designed for rapid and effective identification of visual field impairment owing to glaucoma. The FDT stimulus consists of a bar grid with a low-frequency spatial sinusoidal profile ( 0.25 cycles/degree) subjected to a sinusoidal temporal commutation at a frequency of $25 \mathrm{~Hz}$. FDT is based on the principle of the frequency-doubling illusion by which the subject perceives twice the number of bars really presented. The cells which present a nonlinear response to contrast and which are therefore responsible for this illusion are a subgroup of $\mathrm{M}$ cells.

FDT was performed using the full-threshold program $\mathrm{N}-30$. With this test, target stimuli consisted of individual, 10-degree square, 0.25 cycles/degree, sinusoidal gratings, counter flashing at $25 \mathrm{MHz}$. Targets were in one of the 19 areas within the central $30^{\circ}$ of the visual field. For each visual field, we evaluated the mean defect (MD) and the pattern standard deviation (PSD).

Recently, the use of CDI was proposed to study orbital circulation. ${ }^{19,20}$ This method makes it possible to create a colour-coded vascular map superimposed on a high-resolution B-scan image, thus allowing a quantitative and qualitative assessment of the blood flow in the area examined.

CDI was performed with an AU-580 AsynchronousHitachi analyzer (Hitachi Ltd, Tokyo, Japan) using a $7.5 \mathrm{MHz}$ linear probe. The examination was made by one of us (MC) $)^{21-23}$ in the Ocular Angiology Centre of the Ophthalmology Service of the S Orsola-Malpighi hospital. The patient was placed in a supine position, the exploratory probe was applied to the closed eyelids, and then it was moved until the best signal was obtained for the vessel under examination. The OA was examined in its three segments projecting laterally, above, and medially to the optic nerve shadow, and the CRA was identified in the $2 \mathrm{~mm}$ anterior of the cone shadow of the optic nerve. PCAs were examined laterally or medially to the optic nerve image. The software of the analyzer evaluated the peak systolic velocity (PSV) in centimetre/ $\mathrm{s}$ that is dependent on the beam-vessel angle and the resistance index or Pourcelot's index (RI). The (RI) was calculated using Pourcelot's ratio $(\mathrm{PR}=$ (systolic velocity-diastolic velocity)/systolic velocity). RI is angle independent and is inversely correlated with the resistance of the area perfused by the vessel, so high RI value indicates a compromised perfusion.

All statistical analysis was performed using the FASTAT Version 2 program package (Systat Inc., Evanston, IL, USA) using the Student $t$-test for unpaired data and Bonferroni correction, assuming $P<0.05$ as significant. The Bartlett correlation test was used to assess any correlation between thinning of the RNFL and alterations in the FDT indices with IOP and the haemodynamic indices.

Finally, receiver operating characteristic (ROC) curve analysis was performed to determine the diagnostic sensitivity and specificity of OCT and FDT.

\section{Results}

The OCT testing revealed a thinning of the RNFL in all quadrants in $\mathrm{OH}$ patients as compared to the healthy controls, but only the inferior quadrant was significant $(P<0.045)$.

As regards FDT, the mean MD was worse in $\mathrm{OH}$ patients than in healthy controls and PSD in OH patients was significantly higher $(P<0.044)$ than for healthy controls (Table 2).

ROC analysis revealed that sensitivity and specificity was 85 and $84 \%$ for PSD-FDT, whereas with OCT, a sensitivity of $83 \%$ and specificity of $78 \%$ was only

Table 2 Evaluation of RNFL thickness and FDT indexes in ocular hypertension and healthy control group

\begin{tabular}{lccc}
\hline & Controls & Ocular hypertension & $\mathrm{P}<0.05$ \\
\hline RNFL SUP & $135.083 \pm 10.501$ & $131.250 \pm 15.664$ & 0.138 \\
RNFL INF & $131.750 \pm 10.729$ & $122.250 \pm 14.091$ & 0.045 \\
RNFL TEMP & $83.150 \pm 8.631$ & $79.093 \pm 15.353$ & 0.233 \\
RNFL NAS & $85.663 \pm 7.632$ & $83.150 \pm 8.631$ & 0.273 \\
& & & \\
FDT-MD (dB) & $-1.978 \pm 0.854$ & $-2.034 \pm 0.935$ & 0.666 \\
FDT-PSD (dB) & $1.938 \pm 0.704$ & $3.873 \pm 1.488$ & 0.044 \\
\hline
\end{tabular}

Table 3 Sensitivity and specificity of RNFL thickness evaluation by OCT and FDT visual field to detect glaucomatous defect

\begin{tabular}{lccc}
\hline & Sensitivity (\%) & Specificity (\%) & ROC area \\
\hline RNFL SUP & 57 & 71 & 0.676 \\
RNFL INF & 83 & 78 & 0.824 \\
RNFL TEMP & 64 & 78 & 0.703 \\
RNFL NAS & 64 & 78 & 0.747 \\
MD-FDT & 57 & 71 & 0.505 \\
PSD-FDT & 85 & 84 & 0.923 \\
\hline
\end{tabular}




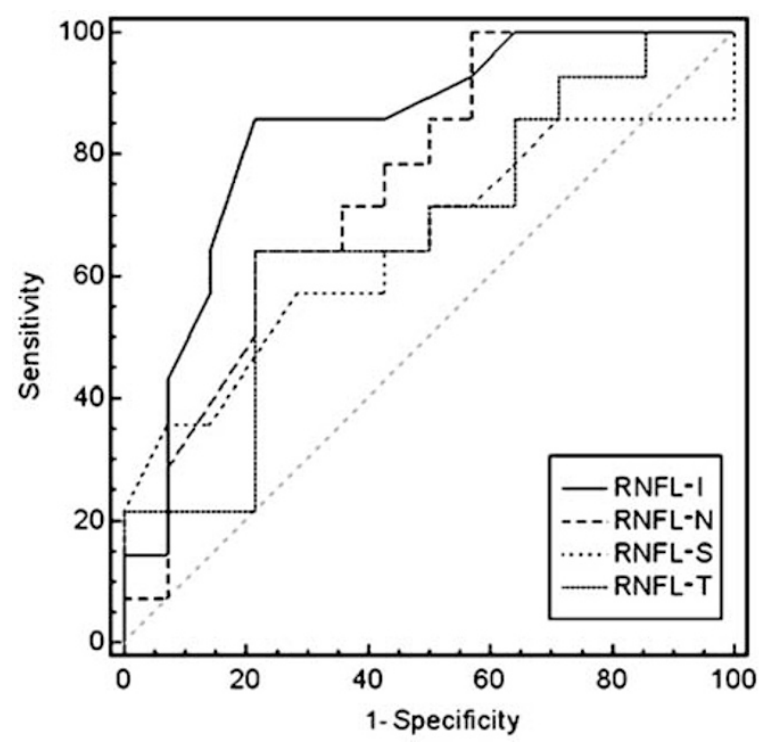

Figure 1 ROC curves and areas for OCT. The ROC curve area for OCT inferior sector thickness was significantly greater than ROC curve area of the other sectors.

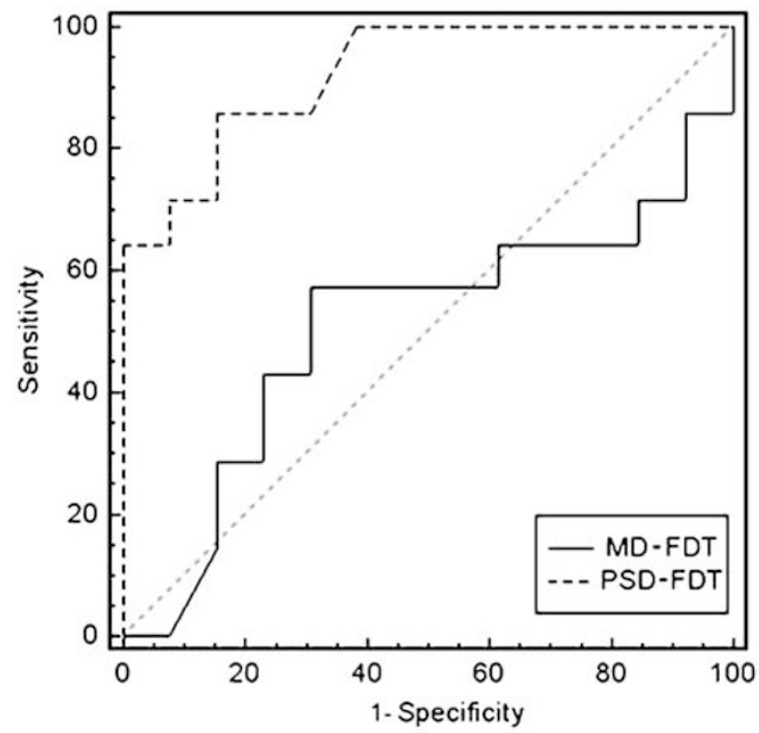

Figure 2 ROC curves and areas for MD-FDT and PSD-FDT The ROC curve area for PSD-FDT was significantly greater than ROC curve area for MD-FDT.

recorded in the inferior RNFL quadrant (Table 3 and Figures 1-3).

$\mathrm{CDI}$ in $\mathrm{OH}$ patients revealed a nonsignificant mean PSV increase of $2 \%$ in the OA, a decrease of $7.3 \%$ in the CRA, and of $4.7 \%$ in the PCAs as compared with the controls. In $\mathrm{OH}$ patients, mean RI was significantly higher in the OA $(P<0.022)$, CRA $(P<0.032)$, and PCAs $(P<0.037)$ than in the healthy controls (Table 4$)$.

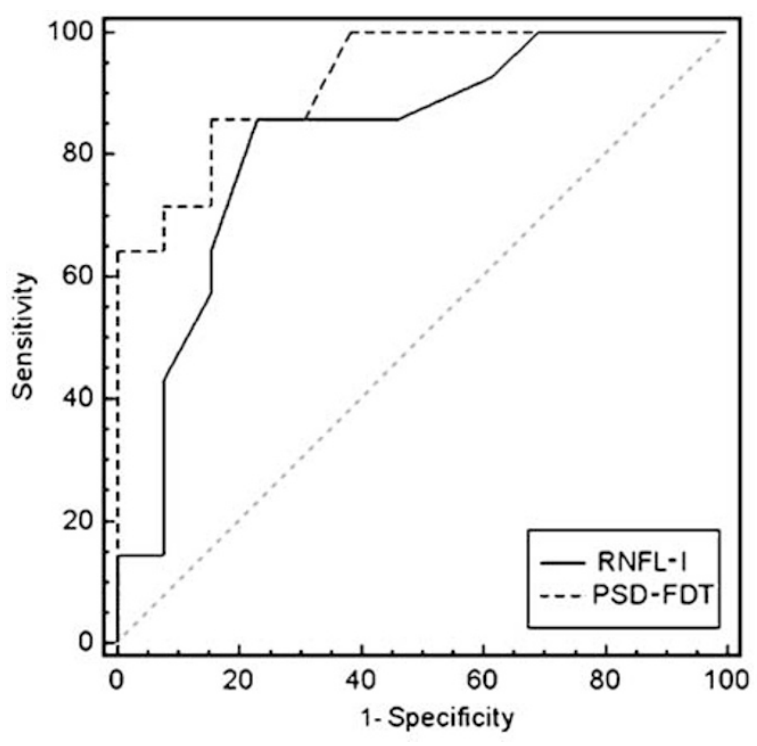

Figure 3 ROC curves and areas for OCT and FDT. The ROC curve area for PSD-FDT is slightly greater than OCT inferior sector nerve fibre layer thickness.

Table 4 Evaluation of CDI indexes in the OA, CRA, and PCAs in ocular hypertension and healthy control group

\begin{tabular}{lrrr}
\hline & \multicolumn{1}{c}{ Controls } & Ocular hypertension & $\mathrm{P}<0.05$ \\
\hline PSV OA & $30.760 \pm 3.745$ & $31.383 \pm 2.553$ & 0.873 \\
RI OA & $0.745 \pm 0.019$ & $0.768 \pm 0.012$ & 0.022 \\
& & & \\
PSV CRA & $13.800 \pm 1.980$ & $12.780 \pm 1.730$ & 0.452 \\
RI CRA & $0.645 \pm 0.019$ & $0.668 \pm 0.012$ & 0.032 \\
& & & \\
PSV PCAs & $14.882 \pm 4.608$ & $14.235 \pm 3.270$ & 0.092 \\
RI PCAs & $0.622 \pm 0.012$ & $0.673 \pm 0.019$ & 0.037 \\
\hline
\end{tabular}

The Bartlett correlation test revealed that the RNFL changes in the inferior quadrant of the optic nerve were correlated with the increase in resistance in the CRA $(P<0.034)$ and the FDT-PSD values to the increase in resistance in the PCAs $(P<0.006)$.

\section{Discussion}

OCT of the RNFL only revealed a significant reduction in thickness in the inferior quadrant of the optic nerve in $\mathrm{OH}$, as compared to healthy controls, with a sensitivity of $83 \%$ and a specificity of $78 \%$. With FDT, there was a significant increase in the PSD index in hypertensive eyes, with a sensitivity of $85 \%$ and a specificity of $84 \%$. Finally, using CDI, a significant increase in the RI was found at the OA, CRA, and PCA levels in patients with 
$\mathrm{OH}$. The Bartlett correlation test showed a correlation between changes in the PSD index, detected with FDT, and the increase in the RI in the PCAs $(P<0.006)$, whereas the thinning of the RNFL inferior quadrant, detected with OCT, was correlated with the increase in RI in the CRA $(P<0.034)$.

Several studies suggest that optic nerve and the RFNL impairment generally can be identified before SAP alterations can be detected. ${ }^{24,25}$ OCT is an optical analogue of an ultrasound B-scan that can create cross-section images of the RNFL in both the axial and lateral dimensions. ${ }^{26}$ As OCT is based on near-infrared interferometry, the thickness measured is not affected by refractive status or axial length of the eye nor by changes in nuclear cataract density. However, cortical cataracts, heavy nuclear cataracts, secondary cataracts, poor vitreous body transparency, and silicon oil in the posterior chamber ${ }^{27,28}$ may affect measurement accuracy.

OCT morphology has shown a good correspondence to histopathological findings. ${ }^{26,29,30}$ The thinning observed in the RNFL inferior quadrant in patients with $\mathrm{OH}$ confirms previous observations, ${ }^{31}$ although with a different percentage incidence that may be owing to the analysis method used. ${ }^{32-35}$ This localized thinning of the RNFL might be an early sign of glaucoma ${ }^{36}$ before any signs of optic nerve disorders or visual field impairment appear. The apparent absence of monochromatic visual field alterations in patients with $\mathrm{OH}$, despite the finding of a very variable incidence of RNFL impairment, may be attributed to the fact that the perimetric methods used are not sufficiently sensitive.

FDT is a highly sophisticated method recently introduced which has been proposed to examine the functionality of the magnocellular ganglion cells. ${ }^{16,37,38}$ Indeed, histopathological experimental studies of the optic nerve in glaucoma patients suggest an early and selective impairment of $\mathrm{M}$ cells, which transport spatial low frequency and temporal high-frequency visual information. ${ }^{39,40}$

FDT is based on the principle of the frequencydoubling illusion by which the subject perceives twice the number of bars really presented. The cells that present a nonlinear response to contrast and are therefore responsible for this illusion are a subgroup of $\mathrm{M}$ cells, called My cells, that overall represent just 3\% of all the retinal ganglion cells. ${ }^{41}$

FDT showed high sensitivity and specificity in detecting functional impairment of a highly selective and limited cell population, thus demonstrating that it is extremely sensitive for the detection of early alterations in ganglion cells. However, the most intriguing question is, why is a thinning in the RNFL and an increase in the PSD index found with FDT in $\mathrm{OH}$ ?
We know that a great deal has been said about the mechanical or vascular aspects in the onset of glaucoma optic neuropathy. Recent studies suggest that the variations in RNFL thickness are correlated with variations in IOP, that is, for each reduction of $1 \mathrm{mmHg}$ in IOP, there is an increase in RNFL of $0.5 \mu \mathrm{m} .{ }^{42}$ These data are, however, much debated since, although they are confirmed by some experimental studies, ${ }^{43,44}$ frequently in single sectors, ${ }^{45}$ in others no optic nerve modifications related to pressure variations have been found. ${ }^{46,47}$

It is still not clear what causes an improvement in the RNFL following a reduction in IOP. One hypothesis explains the increase in RNFL in cases with a reduction in IOP as oedema owing to the sudden reduction in pressure following the filtration operation. A second, mechanical hypothesis suggests that it is related to the reduction in the compression of the lamina cribrosa RNFL and thus the return of the nerve fibre to the shape and thickness before $\mathrm{OH}$. The most interesting factor is, however, that very often, despite the recovery of the RNFL thickness following a reduction in IOP, this increased thickness is not matched by an improvement in the automatic visual field perimetric indices. ${ }^{8,46}$

Our CDI revealed a significant increase in the RI in the CRA which supplies the surface nerve fibre layer and very few or none in the prelaminar region of the optic nerve. ${ }^{48,49}$ These data were significantly correlated with the decrease in thickness in the inferior quadrant of RNFL. Moreover, we found an increased RI in the PCAs supplying the vessels that distribute blood to the laminar and retrolaminar portion of the optic nerve. This was significantly correlated with the increase in the PSD index with the FDT test.

These data confirm previous haemodynamic studies performed on patients with $\mathrm{OH}$ who showed a reduction in blood flow in the temporal quadrants ${ }^{50,51}$ and in the inferior quadrant of the optic nerve. ${ }^{52,53}$ Thus, in the case of $\mathrm{OH}$, the statistical correlation test suggested that anatomical and functional impairment is not related to the increase of the IOP but to ischemic damage at the surface nerve fibre layer, but above all at a laminar and retrolaminar level of the optic nerve.

In conclusion, our study demonstrates that FDT is slightly more sensitive and specific than OCT for highlighting nerve fibre alterations in $\mathrm{OH}$. The relatively minor sensitivity of OCT might depend on the fact that this technique, using coherent light, may be influenced by the opacity of the cornea, lens, and the vitreous humor.

We think that these two investigation techniques are complementary and that the study of the RNFL thickness with OCT and the study of the ganglion cells function with FDT promises to be very useful in identifying patients who may develop a glaucoma. This would 
make possible early drug treatment, not only to reduce IOP but also to improve the blood flow to the optic nerve.

\section{Acknowledgements}

This work was supported in part through the University of Bologna (ECC-MIUR ex-60\%), in part from a donation of the Fondazione Banca del Monte di Bologna e Ravenna, and in part through a gift of the Fondazione Cassa di Risparmio di Bologna.

\section{References}

1 Quigley HA, Addicks EM. Quantitative studies of retinal fiber layer defects. Arch Ophthalmol 1982; 100: 807-814.

2 Quigley HA, Miller NR, George T. Clinical evaluation of nerve fiber layer atrophy as an indicator of glaucomatous optic nerve damage. Arch Ophthalmol 1980; 98: 1564-1571.

3 Dreher AW, Reter K, Weinreb RN. Spatially resolved birefringence of the retinal nerve fiber layer assessed with a retinal elipsometer. Appl Opt 1992; 31: 3730-3749.

4 Weinreb RN, Shakiba S, Zangwill L. Scanning laser polarimetry to measure the nerve fiber layer of normal and glaucomatous eyes. Am J Ophthalmol 1995; 119: 627-663.

5 Gramer E, Maier H, Messmer EM. A measure for the thickness of the nerve fiber layer and the configuration of the optic disc excavation in glaucoma patients: a clinical study using laser tomographic scanner. Proceedings of the Xth International Perimetric Society Meetings. Kugler Pubblications: Amsterdam, 1993, pp 207-213.

6 Maier R, Serguhn S, Gramer E. Sensitivity and specificity of the Heidelberg retina tomography for nerve fiber bundle defects in glaucoma patients with localized visual field defects: a clinical study. Ophthalmologe 1995; 92: 521-525.

7 Huan D, Swanson EA, Lin CP. Optical coherence tomography. Science 1991; 254: 1178-1181.

8 Puliafito CA, Hee MR, Schuman JS, Fujimoto JG. Optical Coherence Tomography of Ocular Diseases. Slack:, 1996, pp 369-374.

9 Koozekanami D, Roberts C, Katz SE, Herderick EE. Intersession repeatability of macular thickness measurements with Humphrey 2000 OCT. Invest Ophthalmol Vis Sci 2000; 41: 1486-1491.

10 Blumenthal EZ, Williams JM, Wainreb RN. Reproducibility of nerve fiber layer thickness measurements by use of optical coherence tomography. Ophthalmology 2000; 107: 2278-2282.

11 Zangwill LM, Bowd C, Berry CC. Discriminating between normal and glaucomatous eyes using the Heidelberg retina tomograph, Gdx nerve fiber analyzer and optical coherence tomograph. Arch Ophthalmol 2001; 119: 985-993.

12 Bowd C, Zangwill LM, Berry CC. Detecting early glaucoma by assessment of retinal nerve fiber layer thickness and visual function. Invest Ophthalmol Vis Sci 2001; 42: 1993-2003.

13 Williams ZY, Schuman JS, Gamell L, Nemi A, Hertzmark E, Fujimoto JG et al. Optical coherence tomography measurement of nerve fiber layer thickness and the likelihood of a visual field defect. Am J Ophthalmol 2002; 134: 538-546.
14 Sample PA. Short-wavelength automated perimetry: it's role in the clinic and for understanding ganglion cell function. Prog Retina Eye Res 2000; 19: 369-383.

15 Johnson CA. Recent developments in automated perimetry in glaucoma diagnosis and management. Curr Opin Ophthalmol 2002; 2: 77-84.

16 Quigley HA. Identification of glaucoma-related visual field abnormality with screening protocol of frequency doubling technology. Am J Ophthalmol 1998; 125: 819-829.

17 Quigley HA, Dunkelberger GR, Baginski TA, Green WR. Chronic human glaucoma causes selectively greater loss of large optic nerve fibers. Ophthalmology 1988; 95: 357-363.

18 Glovinsky Y, Quigley HA, Dunkelberger GR. Retinal ganglion cells loss is size dependent in experimental glaucoma. Invest Ophthalmol Vis Sci 1991; 32: 484-491.

19 Lieb W, Cohen SM, Merton DA, Shields JA, Mitchell DG, Goldberg BB. Color Doppler imaging of the eye and orbit technique and normal vascular anatomy. Arch Ophthalmol 1991; 109: 527-531.

20 Guthoff RF, Berger RW, Winkler P, Helmke K, Chumbley CC. Doppler ultrasonography of the ophthalmic and central retinal vessels. Arch Ophthalmol 1991; 109: 532-536.

21 Cellini M, Caramazza R. Color Doppler imaging of ocular blood flow after topical Ketanserin. Ophthalmologica 1999; 213: $286-289$.

22 Cellini M, Possati GL, Profazio V, Sbrocca M, Caramazza N, Caramazza R. Color Doppler imaging and plasma levels of endothelin-1 in low-tension glaucoma. Acta Ophthalmol Scand Suppl 1997; 224: 11-13.

23 Cellini M, Possati GL, Caramazza N, Caramazza R. Colour Doppler analysis of choroidal circulation in chronic open angle glaucoma. Ophthalmologica 1996; 210: 200-201.

24 Hart Jr WM, Yablonski M, Kass MA, Becker B. Quantitative visual field and optic disc correlates early in glaucoma. Arch Ophthalmol 1978; 96: 2209-2211.

25 Zeyen TG, Caprioli J. Progression of disc and field damage in early glaucoma. Arch Ophthalmol 1993; 111: 62-65.

26 Huang D, Swanson EA, Lin CP. Optical coherence tomography. Science 1991; 254: 1178-1181.

27 Schuman JS, Pedut-Kloizman T, Hertzmark E. Reproducibility of nerve fiber layer thickness measurements using optical coherence tomography. Ophthalmology 1996; 103: $1889-1898$.

28 Mastropasqua L, Carpineto P, Ciancaglini M. Reproducibility of nerve fiber layer thickness measurements using optical coherence tomography in silicone oil-filled eyes. Ophthalmologica 2001; 215: 91-96.

29 Toth CA, Birngruber R, Boppart SA. Argon laser retinal lesion evaluated in vivo by optical coherence tomography. Am J Ophthalmol 1997; 123: 188-198.

30 Hee MR, Izatt JA, Swanson EA. Optical coherence tomography of the human retina. Arch Ophthalmol 1995; 113: 325-332.

31 Bowed C, Weinreb RN, Williams JM, Zangwill LM. The retinal nerve fiber layer thickness in ocular hypertensive, normal and glaucomatous eyes with optical coherence tomography. Arch Ophthalmol 2000; 118: 22-26.

32 Iester M, Broadway DC, Mikelberg FS, Drance SM. A comparison of healthy, ocular hypertensive and glaucomatous optic disc topographic parameters. J Ophthalmol 1997; 6: 363-370.

33 Schwartz B, Takamoto T. Measurements of retinal fiber layer thickness and its functional correlations with the visual field. Bull Soc Belge Ophtalmol 1992; 244: 61-72. 
34 Anton A, Zangwill L, Endadi A, Weinreb RN. Nerve fiber layer measurements with scanning laser polarimetry in ocular hypertension. Am J Ophthalmol 1997; 115: 331-334.

35 Caprioli J. The contour of the juxtapapillary nerve fiber layer in glaucoma. Ophthalmology 1990; 97: 358-365.

36 Quigley HA, Katz J, Derick RJ, Gilbert D, Sommer A. An evaluation of optic disc and nerve fiber layer examinations in monitoring progression of early glaucoma damage. Ophthalmology 1992; 99: 19-28.

37 Johnson CA, Samuels SJ. Screening for glaucomatous visual field loss with frequency-doubling perimetry. Invest Ophthalmol Vis Sci 1997; 38: 413-425.

38 Burnstein Y, Ellish NJ, Magbalon M, Higginbotham EJ. Comparison of frequency-doubling perimetry with Humphrey visual field analysis in a glaucoma practice. Am J Ophthalmol 2000; 3: 328-333.

39 Asai T, Katsumori N, Mizokami K. Retinal ganglion cell damage in human glaucoma, 2: studies on damage pattern. Nippon Ganka Gakkai Zasshi 1987; 91: 1204-1213.

40 Weber AJ, Chen H, Kaufman PL, Hubbard WC. Effects of elevated IOP on cell size, density and number in the primate lateral geniculate nucleus (LGN). ARVO abstracts. Invest Ophthalmol Vis Sci 1997; 38(4, Suppl): 159.

41 Kaplan E, Shapley RM. X and Y cells in the lateral geniculate nucleus of macaque monkeys. J Physiol 1982; 330: 125-143.

42 Aydin A, Wollstein G, Price LL, Fujimoto J, Schuman JS. Optical coherence tomography assessment of retinal nerve fiber layer thickness changes after glaucoma surgery. Ophthalmology 2003; 110: 1506-1511.

43 Quigley HA. Childhood glaucoma: results with trabeculectomy and study of reversible cupping. Ophthalmology 1982; 89: 219-226.

44 Sogano S, Tomita G, Kitazawa Y. Changes in retinal fiber layer thickness after reduction of intraocular pressure in chronic open angle glaucoma. Ophthalmology 1993; 100: 1253-1258.

45 Kotecha A, Siriwardena D, Fitzke FW. Optic disc changes following trabeculectomy: longitudinal and localisation of change. Br J Ophthalmol 2001; 85: 956-961.

46 Lesk MR, Spaeth GL, Azuara-Blanco A. Reversal of optic disk cupping after glaucoma surgery analyzed with scanning laser tomograph. Ophthalmology 1999; 106: 1013-1018.

47 Topouzis F, Peng F, Kotas-Neuman R. Longitudinal changes in optic disc topography of adult patients after trabeculectomy. Ophthalmology 1999; 106: 1147-1151.

48 Lieberman MF, Maumenee AE, Green WR. Histologic studies of the vasculature of the anterior optic nerve. Am J Ophthalmol 1976; 82: 405-423.

49 Cioffi AG, Van Buskirk EM. Microvasculature of the anterior optic nerve. Surv Ophthalmol 1994; 38: S107-S116.

50 Piltz-Seymour JR, Grunwald JE, Hariprasad SM, DuPont J. Optic nerve blood flow is diminished in eyes of primary open angle glaucoma suspects. Am J Ophthalmol 2001; 132: 63-69.

51 Nicolela MT, Drance SM, Rankin SJA, Buckley AR, Walman BE. Color Doppler imaging in asymmetric glaucoma patients with unilateral visual field loss. Am J Ophthalmol 1996; 119: 685-693.

52 Michelson G, Langhans MJ, Harazny J, Dichtl A. Visual field defect and perfusion of the juxtapapillary retina and the neuroretinal rim area in primary open angle glaucoma. Graefes Arch Clin Exp Ophthalmol 1998; 226: 80-85.

53 Zink JM, Grunwald JE, Plitz-Seymour J, Staii A, DuPont J. Association between lower optic nerve laser Doppler blood volume measurements and glaucomatous visual field progression. Br J Ophthalmol 2003; 87: 1487-1491. 